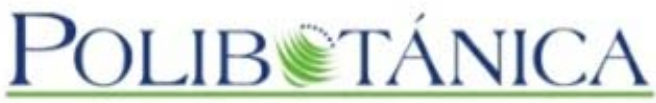

\section{Polibotánica}

ISSN electrónico: 2395-9525

polibotanica@gmail.com

Instituto Politécnico Nacional

México

http://www.polibotanica.mx

\title{
EQUILIBRIO HIGROSCÓPICO EN SEMILLAS FORESTALES DE ZONAS ÁRIDAS Y SU EFECTO EN LA CALIDAD FISIOLÓGICA
}

\section{HYGROSCOPIC EQUILIBRIUM IN FOREST SEEDS OF ARID ZONE AND ITS EFFECT ON PHYSIOLOGICAL QUALITY}

Antonio-Bautista, A., M.E. Vázquez-Badillo, M.H. Reyes-Valdés, C. Flores-López, E.J. Cruz-Gutiérrez y D.U. González-Uribe EQUILIBRIO HIGROSCÓPICO EN SEMILLAS FORESTALES DE ZONAS ÁRIDAS Y SU EFECTO EN LA CALIDAD FISIOLÓGICA

HYGROSCOPIC EQUILIBRIUM IN FOREST SEEDS OF ARID ZONE AND ITS EFFECT ON PHYSIOLOGICAL QUALITY

\section{POLIBETANICA} Instituto Politécnico Nacional
Núm. 50: 67-81 México. Agosto 2020

DOI: $10.18387 /$ polibotanica.50.5

(c) (†) Este es un artículo de acceso abierto bajo la licencia Creative Commons 4.0 Atribución-No Comercial (CC BY-NC 4.0 Internacional). 


\section{EQUILIBRIO HIGROSCÓPICO EN SEMILLAS FORESTALES DE ZONAS ÁRIDAS Y SU EFECTO EN LA CALIDAD FISIOLÓGICA}

\section{HYGROSCOPIC EQUILIBRIUM IN FOREST SEEDS OF ARID ZONE AND ITS EFFECT ON PHYSIOLOGICAL QUALITY}

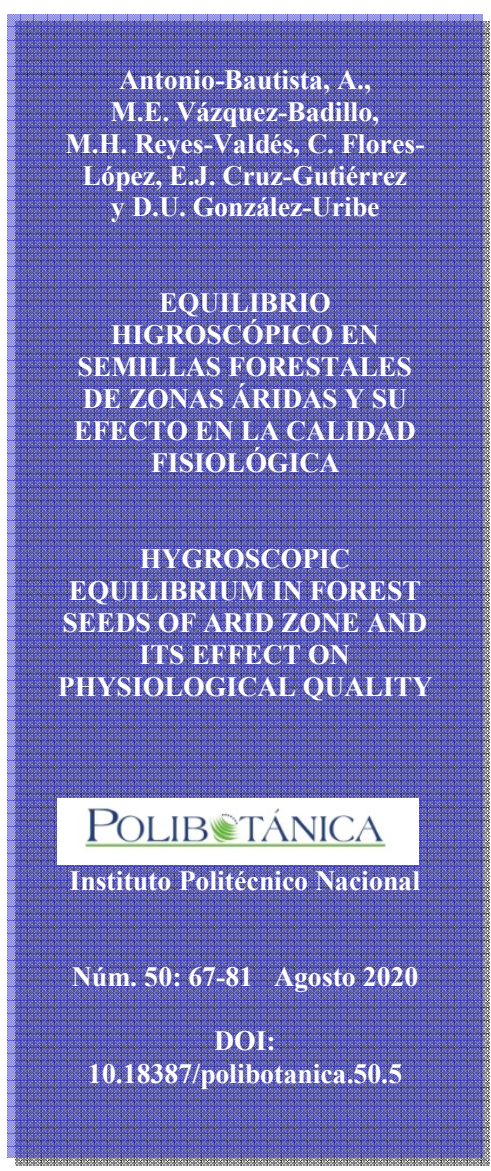

\author{
A. Antonio-Bautista \\ M.E. Vázquez-Badillo / marioe.vazquez@hotmail.com \\ M.H. Reyes-Valdés \\ Departamento de Fitomejoramiento. \\ Universidad Autónoma Agraria Antonio Narro \\ Calzada Antonio Narro 1923, Buenavista, 25315 Saltillo, Coahuila, México.
}

C. Flores-López

Departamento de Forestal-UAAAN.

E.J. Cruz-Gutiérrez

Centro Nacional de Recursos Genéticos-Instituto Nacional de Investigaciones Agrícolas y Pecuarias. Boulevard de la biodiversidad 400, Rancho las Cruces, 47600 Tepatitlán de Morelos, Jalisco, México.

D.U. González-Uribe Departamento de Estadística y Cálculo-UAAAN.

RESUMEN: Las semillas forestales son el material mayormente utilizado para la producción masiva de plantas, las especies de zonas áridas no producen semilla todos los años, es necesario disponer de germoplasma en cantidad y calidad que permitan la obtención de nuevos individuos. Conocer la humedad en equilibrio, permite establecer los tiempos de almacenamiento seguro, durante un tiempo determinado. El objetivo de este trabajo fue evaluar el efecto del contenido de humedad de equilibrio sobre la calidad fisiológica en semillas de Agave lecheguilla Torr., Lippia graveolens Kunt., y Nolina cespitifera Trel., durante el almacenamiento. Las semillas fueron almacenadas por un periodo de 90 días bajo 12 ambientes, se evaluaron a $0,15,30,60$ y 90 días, con cuatro repeticiones de 100 semillas, las pruebas fisiológicas y humedad de la semilla fueron realizadas utilizando procedimientos internacionales de la Asociación Internacional de Análisis de Semillas (International Seed Testing Association) (ISTA, 2014). Las variables evaluadas fueron humedad de la semilla (HS), índice de velocidad de germinación (IVG), porcentaje de plántulas normales $(\mathrm{PN})$, porcentaje de plántulas anormales(PA), porcentaje de semillas muertas (SM) y porcentaje de semillas duras (SD), El diseño experimental fue factorial con arreglo de tratamientos completamente al azar. Los resultados mostraron que las semillas almacenadas a $60-75 \%$ de humedad relativa y $5-15^{\circ} \mathrm{C}$ no perdieron de manera significativa su capacidad germinativa, la $\mathrm{HS}$ osciló entre $12-13 \%$, los valores más altos de HS se obtuvieron a $80-85 \%$ de humedad relativa y en respuesta el porcentaje de PN disminuyó de manera considerable entre 5.0 y $18.5 \%$. En las tres especies en estudio la humedad en equilibrio ésta fuertemente afectada por las condiciones de almacenamiento, alta humedad relativa y temperatura ocasionan humedad de equilibrio alta, afectando de manera negativa la calidad fisiológica y ocasionando un rápido deterioro.

Palabras clave: Almacenamiento, Germinación, Deterioro, Humedad de Equilibrio, Longevidad. 
ABSTRACT: Forest seeds are the material mostly used for the mass production of plants; the species of arid zones do not produce seed every year, it is necessary to have germplasm in quantity and quality that allow obtaining new individuals. Knowing the moisture in equilibrium, allows establishing safe storage times, for a certain time. The objective of this work was to evaluate the effect of the moisture content of Balance on the physiological quality in seeds of Agave lecheguilla Torr., Lippia graveolens Kunt., and Nolina cespitifera Trel., during storage. The seeds were stored for a period of 90 days under 12 environments, were evaluated at 0,15 , 30, 60 and 90 days, with four repetitions of 100 seeds, physiological tests and seed moisture were performed using international procedures of the Association International Seed Analysis Association (ISTA, 2014). The variables evaluated were seed moisture (HS), germination speed index (IVG), percentage of normal seedlings (PN), percentage of abnormal seedlings (PA), percentage of dead seeds (SM) and percentage of hard seeds (SD), The experimental design was factorial according to completely randomized treatments. The results showed that the seeds stored at $60-75 \%$ relative humidity and $5-15{ }^{\circ} \mathrm{C}$ did not significantly lose their germination capacity, the HS ranged between $12-13 \%$, the highest HS values were are obtained at $80-85 \%$ relative humidity and in response, the percentage of PN decreased considerably between 5.0 and $18.5 \%$. In the three species under study, equilibrium moisture is strongly affected by storage conditions, high relative humidity and temperature cause high equilibrium humidity, negatively affecting physiological quality and causing rapid deterioration.

Key word: Storage, Germination, Deterioration, Equilibrium of Moisture, Longevity.

\section{INTRODUCCIÓN}

A nivel mundial los desiertos ocupan un $40 \%$ de la superficie, es decir 33.7 millones de $\mathrm{km}^{2}$. México posee una superficie total de 2 millones aproximadamente, de los cuales las zonas áridas y semiáridas ocupan entre 50 y $60 \%$ del territorio (González, 2012). Se caracterizan por presentar una gran antidad de especies forestales clasificadas como no maderables y con gran potencial económico, por el uso de fibras y componentes fitoquímicos que de éstas especies se obtienen (Tropicos, 2019).

Sin embargo, las poblaciones naturales de especies forestales no maderables de zonas áridas se han visto disminuidas, tanto por el aprovechamiento como por el impacto de desastres naturales, incendios forestales, sequía, plagas y enfermedades (Doria, 2010).

Las semillas son consideradas como la fuente más importantes de germoplasma primario y hasta el momento constituyen el material mayormente utilizado para la producción masiva de plantas, con fines de reforestación o establecimiento de plantaciones (Sáenz et al., 2011).

La forma de propagación de especies forestales no maderables de zonas áridas es principalmente por semilla, sin embargo, no mantienen una producción anual constante, por lo que hace inevitable contar con germoplasma en cantidad y calidad necesaria para abastecer los programas de producción de planta (Iriondo, 2001). Por tanto es necesario almacenarlas y conservarlas hasta el momento de su utilización sin que se pierdan sus atributos de calidad genética, física, fisiológica y sanitaria (Romero y Pérez, 2016).

Las semillas de especies de zonas áridas y semiáridas se caracteriza por presentar un tipo de latencia exógena, dicha latencia pudiera deberse principalmente a propiedades físicas y químicas de las cubiertas seminales (Baskin y Baskin, 2004), así como la latencia endógena que es determinada por características anatómicas, morfológicas y fisiológicas del propio embrión (latencia embrionaria) que impide el paso de agua para iniciar el proceso de germinación (Doria, 2010).

Las semillas almacenadas están sujetas a diversos cambios uno de ellos es la pérdida de la calidad fisiológica lo que ocasiona su deterioro, sin embargo, cuando el almacenamiento es 
llevado a cabo bajo condiciones favorables, se pueden almacenar por largos periodos sin que esto represente algún problema, los factores más importantes que afectan el almacenamiento de semillas son la humedad relativa, la temperatura y la condición de la semilla (Salazar et al., 2011).

El contenido de humedad de equilibrio (CHE) o equilibrio higroscópico, es el contenido de humedad que la semilla alcanza, cuando se almacena durante un tiempo en condiciones de temperatura y humedad relativa (Mahecha Godoy, 2011). El conocimiento de la humedad en equilibrio de las semillas bajo condiciones ambientales, permite establecer los tiempos seguros de almacenamiento durante un tiempo determinado (Vega et al., 2006).

Debido a que no se cuenta con información necesaria, que proporcione las condiciones óptimas de almacenamiento para el resguardo a corto, mediano y largo plazo, en semillas de especies forestales no maderables de zonas áridas, se plantea el siguiente objetivo, evaluar el efecto de la humedad en equilibrio en semillas Agave lecheguilla Torr., Lippia graveolens Kunt., y Nolina cespitifera Trel., en la calidad fisiológica, durante el almacenamiento.

\section{MATERIAL Y MÉTODOS}

La investigación se realizó en el Laboratorio de Almacenamiento de Granos y Semillas del Centro de Capacitación y Desarrollo de Tecnología de Semillas (CCDTS) de la UAAAN, situada, en Buenavista, Saltillo, Coahuila. Se utilizaron semillas de agave (Agave lecheguilla Torr.), orégano (Lippia graveolens Kunt.), y cortadillo (Nolina cespitifera Trel.), especies forestales no maderables de zonas áridas de importancia económica ya que de ellas se obtienen fibras y componentes fitoquímicos, se obtuvieron de lotes semilleros de poblaciones naturales, del sureste de Coahuila, colectadas en 2016, verificando que todas las semillas contaran con la madurez fisiológica adecuada, estuviera bien conformada y en un estado masoso, siendo éste último un indicativo que la semilla está completamente madura.

Las semillas se almacenaron por un periodo de 90 días en 12 ambientes que resultaron de la combinación de tres temperaturas $\left(5,15\right.$ y $\left.30^{\circ} \mathrm{C}\right)$ y cuatro niveles de humedad relativa $(60,75$, 80 y $85 \%$ ), éstas humedades relativas se obtuvieron de manera artificial con soluciones sobresaturadas de acuerdo a Winston y Bates (1960).

Se utilizaron cuatro repeticiones de 100 semillas de cada especie en estudio, resultando 240 unidades experimentales. Se colocaron en mallas de tela perforadas y estas a su vez se distribuyeron al azar en cámaras de plástico que conformaban cada uno de los ambientes del almacén. Se sellaron con cinta adhesiva, para que las soluciones actuaran correctamente, las muestras fueron evaluadas a los $0,15,30,60$ y 90 días, la evaluación inicial se realizó a cero días de almacenamiento con el fin de conocer el contenido de humedad de las semillas y el estado fisiológico antes de ser sometidas al periodo de almacenamiento. Las semillas almacenadas se sometieron a una prueba de humedad en contenedores de aluminio en una estufa de secado a temperatura de $103{ }^{\circ} \mathrm{C}$ durante $17 \mathrm{~h}$; además, se realizó un análisis de germinación, el cual consistió en colocar las semillas en contenedores de plástico de 20 X 15 X $5 \mathrm{~cm}$ que contenían sustrato estéril tipo peatmoss con características deseables para realizar la prueba de germinación ISTA (2014). Las semillas se distribuyeron al azar sobre la superficie del sustrato previamente humedecido y fueron llevadas a una cámara germinadora Seed Buro con condiciones controladas de humedad $(80 \%)$, temperatura de $22 \pm 2.5^{\circ} \mathrm{C}$, y con $12 \mathrm{~h}$ de luz y $8 \mathrm{~h}$ de obscuridad. Se aplicaron riegos cada tercer día con agua corriente, para que el sustrato tuviera las condiciones óptimas de humedad y la semilla iniciara el proceso de germinación. Se realizaron dos conteos, el primero a los 14 días y el segundo a los 21 días después de la siembra, éstas pruebas se realizaron utilizando procedimientos internacionales de la Asociación Internacional de Análisis de Semillas (International Seed Testing Association) (ISTA, 2014). 
Las variables a evaluar fueron: humedad de la semilla (HS), índice de velocidad de germinación (IVG) de acuerdo a Maguire (1962), porcentaje de plántulas normales (PN) se definió como la relación entre el número de semillas germinadas que tenían todas sus estructuras esenciales y el número de semillas plantadas, porcentaje de plántulas anormales(PA) se contaron aquellas plántulas que carecían de una o más de sus estructuras esenciales, porcentaje de semillas muertas (SM): el conteo incluyó todas aquellas semillas que eran blandas y absorbieron agua, pero no produjeron plántulas y porcentaje de semillas duras (SD) se contaron todas las semillas que no absorbieron agua y que permanecieron impermeables al final del ensayo. El diseño experimental fue factorial (factor $\mathrm{A}=$ tiempo, $\mathrm{B}=$ humedad relativa y $\mathrm{C}=$ temperatura) con arreglo de tratamientos completamente al azar. Los datos se sometieron a análisis de varianza, y cuando hubo diferencias, se realizó una comparación de medias mediante prueba de Tukey ( $\mathrm{p}=$ 0.05), con el software estadístico R Core Team (RCT, 2017).

\section{RESUltados}

\section{Humedad de la semilla}

En la figura 1 se observa que el contenido de la humedad de la semilla (HS) está fuertemente afectada por la humedad relativa del ambiente (HR), en las tres especies en estudio HS fue mayor cuando las semillas estuvieron almacenadas en condiciones de alta HR (85\%).
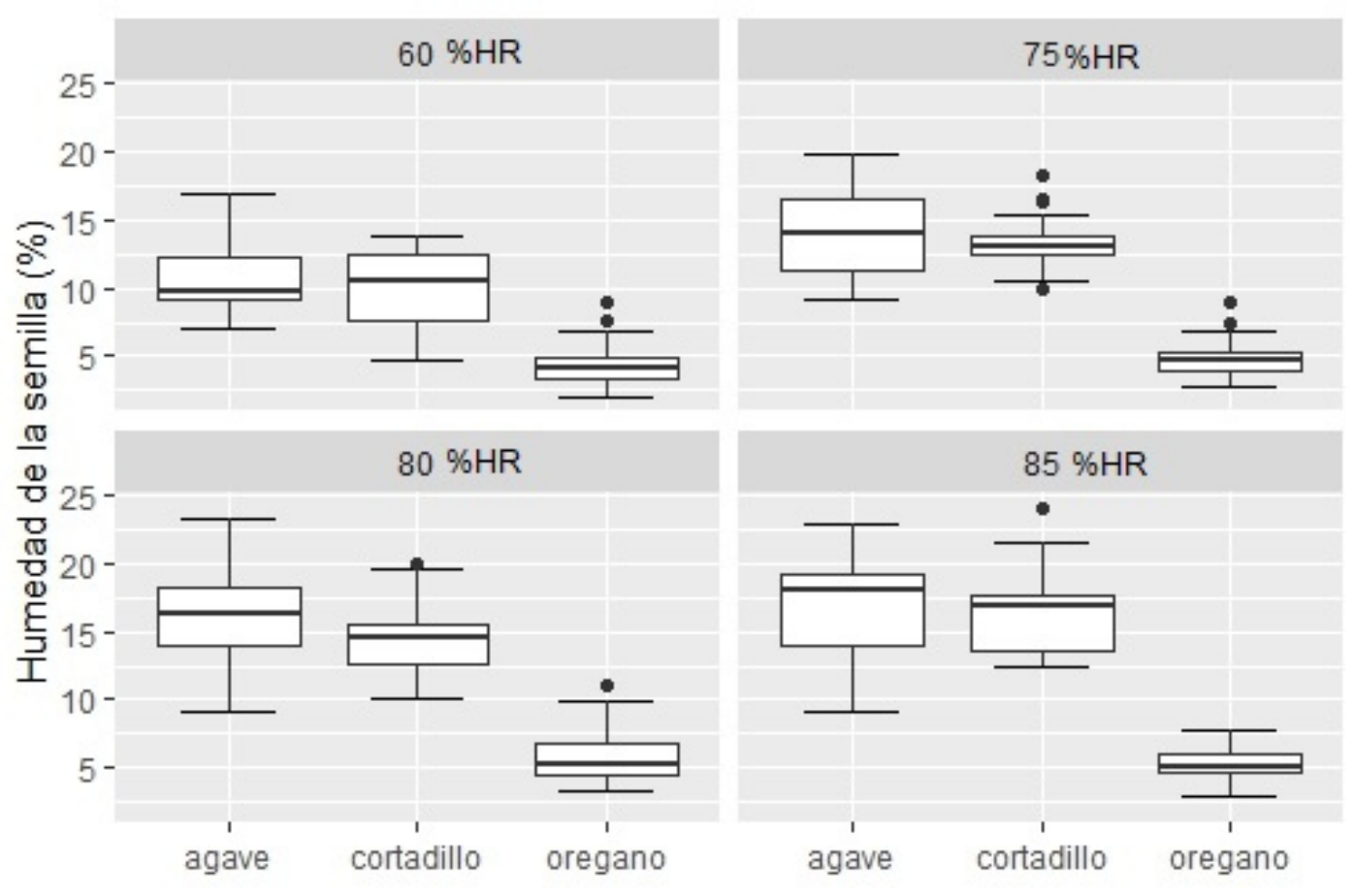

Fig. 1. Humedad de la semilla de agave (Agave lecheguilla Torr.), orégano (Lippia graveolens Kunt.) y cortadillo (Nolina cespitifera Trel.), almacenadas en diferentes niveles de humedad relativa.

La humedad de la semilla (HS) de las tres especies en estudio, fue mayor cuando se almaceron en condiciones de baja temperatura $\left(5^{\circ} \mathrm{C}\right)$ y menor cuando la temperatura del ambiente fue de $25^{\circ} \mathrm{C}$ (fig. 2). 

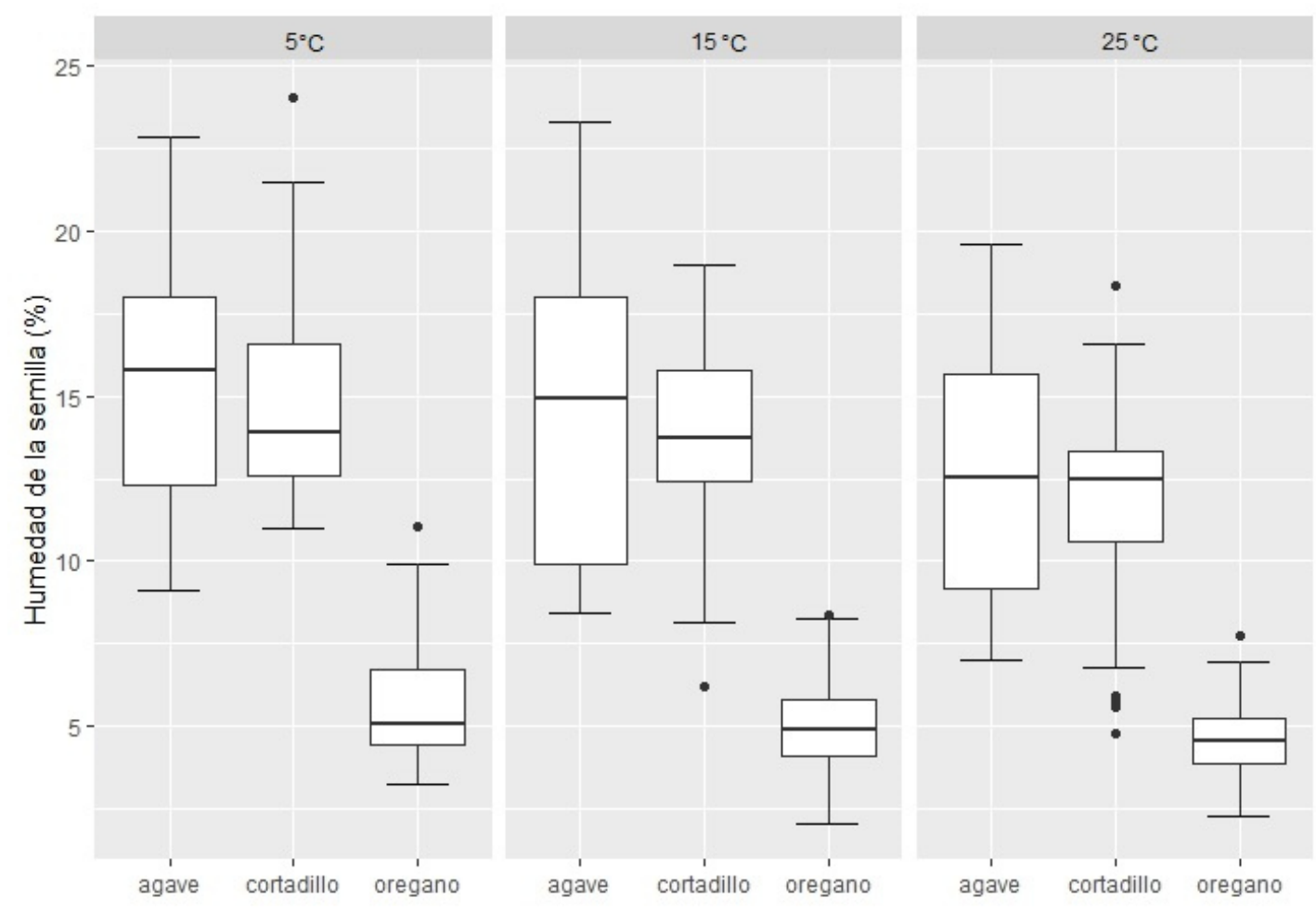

Fig. 2. Humedad de la semilla de agave (Agave lecheguilla Torr.), orégano (Lippia graveolens Kunt.), y cortadillo (Nolina cespitifera Trel.), almacenadas a tres temperaturas.

En la figura 3, se observa para las tres especies en estudio, que a medida que pasa el tiempo la humedad de la semilla aumenta y se estabilizó en el tercer muestreo es decir entra en equilibrio con el ambiente a partir de los 30 días. 

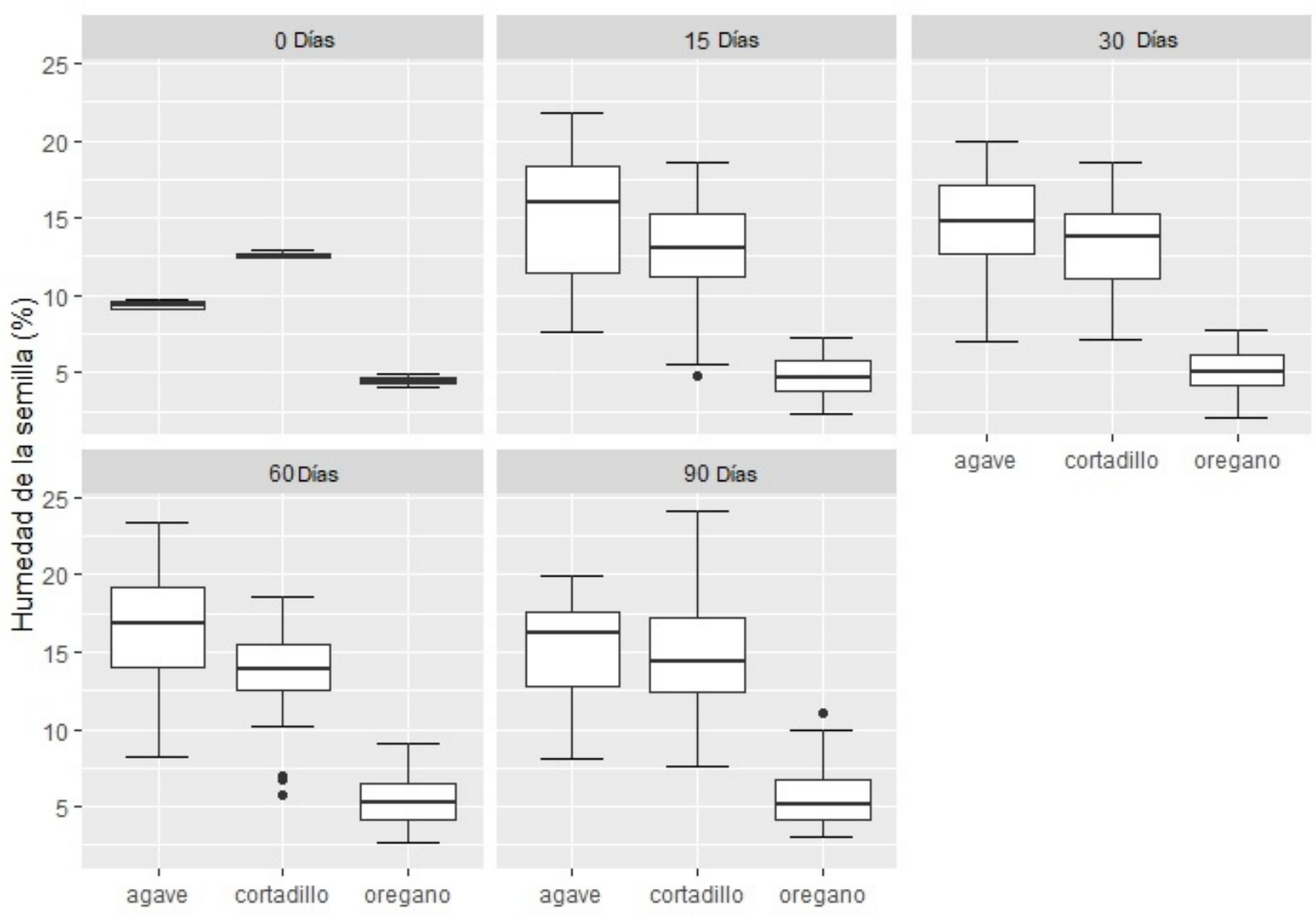

Fig. 3. Humedad de la semilla de agave (Agave lecheguilla Torr.), orégano (Lippia graveolens Kunt.) y cortadillo (Nolina cespitifera Trel.), almacenadas a 90 días.

En A. lecheguilla Torr., el contenido de humedad de la semilla, se mantiene en valores bajos (12-13\%) en condiciones de $60 \%$ de humedad relativa y $5{ }^{\circ} \mathrm{C}$, alcanzando los valores más altos de HS $(20.75 \%)$ en condiciones de humedad relativa $85 \%$ (cuadro 1 ).

Para semillas de $L$. graveolens Kunt (cuadro 2), en las diferentes condiciones de humedad relativa y temperatura se mantuvieron en valores entre 3.8 y $7.5 \%$ de humedad de la semilla durante los 90 días de almacenamiento.

El comportamiento en el contenido de humedad de la semilla en $N$. cespitifera Trel, muestra que en condiciones de humedad relativa baja (60\%) los valores observados oscilaron entre $8.3 \mathrm{y}$ $12.6 \%$ (cuadro 3 ).

\section{Variables fisiológicas}

En semillas de A. lecheguilla Torr. Se observa que en humedades relativas bajas (60\%) los valores de IVG y PN fueron poco afectadas con respecto al muestreo inicial, sin embargo, en humedades relativas altas, los valores disminuyeron (37.16\%) después de 90 días de almacenamiento (cuadro 1). 
Cuadro 1. Comparación de medias de Tukey $(\mathrm{p}>0.05)$ para las variables evaluadas en semillas de agave (Agave lecheguilla Torr.) en cinco muestreos bajo cuatro niveles de humedad relativa.

\begin{tabular}{|c|c|c|c|c|c|c|c|}
\hline Días & HR \% & HS & IVG & PN & PA & SM & SD \\
\hline \multirow{4}{*}{0} & 60 & $9.37 \mathrm{~h}$ & $5.21 \mathrm{a}$ & $96.00 \mathrm{a}$ & $4.00 \mathrm{~cd}$ & $0.00 \mathrm{e}$ & $0.00 \mathrm{c}$ \\
\hline & 75 & $9.37 \mathrm{~h}$ & $5.21 \mathrm{a}$ & $96.00 \mathrm{a}$ & $4.000 \mathrm{~cd}$ & $0.00 \mathrm{e}$ & $0.00 \mathrm{c}$ \\
\hline & 80 & $9.37 \mathrm{~h}$ & $5.21 \mathrm{a}$ & $96.00 \mathrm{a}$ & $4.00 \mathrm{~cd}$ & $0.00 \mathrm{e}$ & $0.00 \mathrm{c}$ \\
\hline & 85 & $9.37 \mathrm{~h}$ & $5.20 \mathrm{a}$ & $96.00 \mathrm{a}$ & $4.00 \mathrm{~cd}$ & $0.00 \mathrm{e}$ & $0.00 \mathrm{c}$ \\
\hline \multirow{4}{*}{15} & 60 & $10.20 \mathrm{gh}$ & $4.73 \mathrm{abc}$ & $86.50 \mathrm{abc}$ & $5.66 \mathrm{~cd}$ & $6.83 \mathrm{de}$ & $1.00 \mathrm{abc}$ \\
\hline & 75 & $12.67 \mathrm{fg}$ & $4.55 \mathrm{abcd}$ & $82.91 \mathrm{abcd}$ & $6.33 \mathrm{~cd}$ & $10.75 \mathrm{de}$ & $0.00 \mathrm{c}$ \\
\hline & 80 & 17.20 bcde & 3.94 bcdef & 70.33 bcde & $10.16 \mathrm{~cd}$ & $19.50 \mathrm{cde}$ & $0.00 \mathrm{c}$ \\
\hline & 85 & $19.63 \mathrm{ab}$ & $3.68 \mathrm{cdefg}$ & $64.66 \mathrm{cdef}$ & $12.33 \mathrm{bcd}$ & $23.00 \mathrm{cde}$ & $0.00 \mathrm{c}$ \\
\hline \multirow{4}{*}{30} & 60 & $11.00 \mathrm{gh}$ & $4.90 \mathrm{ab}$ & $89.16 \mathrm{ab}$ & $6.75 \mathrm{~cd}$ & $1.66 \mathrm{e}$ & $2.50 \mathrm{abc}$ \\
\hline & 75 & $15.03 \mathrm{ef}$ & $4.34 \mathrm{abcd}$ & 79.00 bcde & $6.33 \mathrm{~cd}$ & $11.33 \mathrm{de}$ & $3.33 \mathrm{ab}$ \\
\hline & 80 & $15.56 \mathrm{de}$ & $3.01 \mathrm{efgh}$ & $55.08 \mathrm{efgh}$ & $3.33 \mathrm{~d}$ & $41.58 b c$ & $0.00 \mathrm{c}$ \\
\hline & 85 & $16.71 \mathrm{cde}$ & 3.43 defgh & $61.41 \mathrm{defg}$ & $7.91 \mathrm{~cd}$ & $30.33 \mathrm{bcd}$ & $0.33 \mathrm{bc}$ \\
\hline \multirow{4}{*}{60} & 60 & $11.20 \mathrm{gh}$ & 4.09 abcde & 72.58 abcde & $12.75 \mathrm{bcd}$ & $14.66 \mathrm{de}$ & $0.00 \mathrm{c}$ \\
\hline & 75 & $15.65 \mathrm{cde}$ & $3.83 \mathrm{bcdef}$ & $64.50 \mathrm{cdef}$ & $20.75 \mathrm{abc}$ & $11.16 \mathrm{de}$ & $3.66 \mathrm{a}$ \\
\hline & 80 & $17.80 \mathrm{bcd}$ & $1.16 \mathrm{i}$ & $17.66 \mathrm{i}$ & $12.00 \mathrm{bcd}$ & $70.33 \mathrm{a}$ & $0.00 \mathrm{c}$ \\
\hline & 85 & $18.34 \mathrm{abc}$ & $2.34 \mathrm{hi}$ & 39.66 ghi & $12.50 \mathrm{bcd}$ & $47.91 \mathrm{ab}$ & $0.00 \mathrm{c}$ \\
\hline \multirow{4}{*}{90} & 60 & $11.59 \mathrm{gh}$ & $2.86 \mathrm{fgh}$ & $44.00 \mathrm{fgh}$ & $28.33 \mathrm{ab}$ & $27.75 \mathrm{bcd}$ & $0.00 \mathrm{c}$ \\
\hline & 75 & 15.92 cde & $2.92 \mathrm{efgh}$ & $42.58 \mathrm{fgh}$ & $36.33 \mathrm{a}$ & $21.16 \mathrm{cde}$ & $0.00 \mathrm{c}$ \\
\hline & 80 & $16.68 \mathrm{cde}$ & $2.60 \mathrm{gh}$ & $35.66 \mathrm{hi}$ & $36.50 \mathrm{a}$ & $27.75 \mathrm{bcd}$ & $0.00 \mathrm{c}$ \\
\hline & 85 & $20.75 \mathrm{a}$ & $2.33 \mathrm{hi}$ & $37.16 \mathrm{hi}$ & $19.50 \mathrm{abcd}$ & $43.33 \mathrm{bc}$ & $0.00 \mathrm{c}$ \\
\hline
\end{tabular}

Las medias con la misma letra indican que no son significativamente diferentes, HS = Humedad de la semilla, IVG = Índice de velocidad de germinación, PN = plántulas normales, $\mathrm{PA}=$ plántulas anormales, $\mathrm{SM}=$ semillas muertas, $\mathrm{SD}$ $=$ semillas duras.

En el cuadro 2 se puede observar el comportamiento de semillas de L. graveolens Kunt. cuando éstas fueron expuestas a condiciones de alta humedad relativa, las variables IGV y PN presentaron los valores más bajos después del periodo de almacenamiento (1.93) y $(58.00 \%)$ respectivamente. 
Cuadro 2. Comparación de medias de Tukey $(\mathrm{p}>0.05)$ para las variables evaluadas en semillas de orégano (Lippia graveolens Kunt.) en cinco muestreos bajo diferentes humedades relativas.

\begin{tabular}{cclllll}
\hline Días & HR \% & \multicolumn{1}{c}{ HS } & \multicolumn{1}{c}{ IVG } & \multicolumn{1}{c}{ PN } & PA & SM \\
\hline \multirow{4}{*}{0} & 60 & $4.50 \mathrm{cde}$ & $2.95 \mathrm{a}$ & $89.48 \mathrm{a}$ & $4.815 \mathrm{a}$ & $5.70 \mathrm{~g}$ \\
& 75 & $4.50 \mathrm{cde}$ & $2.95 \mathrm{a}$ & $89.48 \mathrm{a}$ & $4.815 \mathrm{a}$ & $5.70 \mathrm{~g}$ \\
& 80 & $4.50 \mathrm{cde}$ & $2.95 \mathrm{a}$ & $89.48 \mathrm{a}$ & $4.815 \mathrm{a}$ & $5.70 \mathrm{~g}$ \\
& 85 & $4.50 \mathrm{cde}$ & $2.95 \mathrm{a}$ & $89.48 \mathrm{a}$ & $4.815 \mathrm{a}$ & $5.70 \mathrm{~g}$ \\
\hline \multirow{3}{*}{15} & 60 & $4.83 \mathrm{bcde}$ & $2.95 \mathrm{a}$ & $89.66 \mathrm{a}$ & $1.00 \mathrm{~b}$ & $8.33 \mathrm{fg}$ \\
& 75 & $4.03 \mathrm{de}$ & $2.70 \mathrm{ab}$ & $82.00 \mathrm{ab}$ & $2.00 \mathrm{ab}$ & $16.00 \mathrm{ef}$ \\
& 80 & $4.81 \mathrm{bcde}$ & $2.53 \mathrm{bc}$ & $77.00 \mathrm{bc}$ & $2.00 \mathrm{ab}$ & $22.00 \mathrm{cde}$ \\
& 85 & $5.21 \mathrm{bcde}$ & $2.51 \mathrm{bcd}$ & $76.33 \mathrm{bc}$ & $1.66 \mathrm{ab}$ & $22.00 \mathrm{cde}$ \\
\hline \multirow{3}{*}{30} & 60 & $3.55 \mathrm{e}$ & $2.35 \mathrm{cde}$ & $71.00 \mathrm{cde}$ & $3.66 \mathrm{ab}$ & $25.33 \mathrm{bcde}$ \\
& 75 & $5.32 \mathrm{bcd}$ & $2.33 \mathrm{cde}$ & $70.66 \mathrm{cde}$ & $2.00 \mathrm{ab}$ & $27.33 \mathrm{bcd}$ \\
& 80 & $5.64 \mathrm{bcd}$ & $2.29 \mathrm{cde}$ & $69.66 \mathrm{cde}$ & $1.33 \mathrm{ab}$ & $29.00 \mathrm{abcd}$ \\
& 85 & $5.79 \mathrm{bc}$ & $2.17 \mathrm{ef}$ & $65.66 \mathrm{ef}$ & $2.66 \mathrm{ab}$ & $31.66 \mathrm{ab}$ \\
\hline \multirow{2}{*}{60} & 60 & $4.72 \mathrm{bcde}$ & $2.50 \mathrm{bcd}$ & $75.33 \mathrm{bcd}$ & $4.00 \mathrm{ab}$ & $20.66 \mathrm{de}$ \\
& 75 & $5.04 \mathrm{bcde}$ & $2.38 \mathrm{cde}$ & $72.00 \mathrm{cde}$ & $2.66 \mathrm{ab}$ & $25.33 \mathrm{bcde}$ \\
& 80 & $6.33 \mathrm{ab}$ & $2.31 \mathrm{cde}$ & $70.33 \mathrm{cde}$ & $1.33 \mathrm{ab}$ & $28.33 \mathrm{bcd}$ \\
& 85 & $5.31 \mathrm{bcd}$ & $2.18 \mathrm{ef}$ & $66.00 \mathrm{def}$ & $2.66 \mathrm{ab}$ & $31.33 \mathrm{abc}$ \\
\hline & 60 & $4.00 \mathrm{de}$ & $2.29 \mathrm{cde}$ & $69.33 \mathrm{cde}$ & $3.33 \mathrm{ab}$ & $27.33 \mathrm{bcd}$ \\
& 75 & $4.82 \mathrm{bcde}$ & $2.20 \mathrm{def}$ & $66.33 \mathrm{def}$ & $3.33 \mathrm{ab}$ & $30.33 \mathrm{abc}$ \\
& 80 & $7.53 \mathrm{a}$ & $2.14 \mathrm{ef}$ & $64.66 \mathrm{ef}$ & $3.33 \mathrm{ab}$ & $32.00 \mathrm{ab}$ \\
& 85 & $5.88 \mathrm{abc}$ & $1.93 \mathrm{f}$ & $58.00 \mathrm{f}$ & $3.66 \mathrm{ab}$ & $38.33 \mathrm{a}$ \\
\hline
\end{tabular}

Las medias con la misma letra indican que no son significativamente diferentes, HS=Humedad de la semilla, IVG = Índice de velocidad de germinación, $\mathrm{PN}=$ plántulas normales, $\mathrm{PA}=$ plántulas anormales, $\mathrm{SM}=$ semillas muertas.

Respecto al porcentaje de PA y SM en las tres especies en estudio aumentaron cuando fueron almacenadas en condiciones de alta humead relativa (85\%), presentando valores $70 \%$ después de los 90 días de almacenamiento.

Las semillas de $N$. cespitifera Trel., mostraron un aumento SD ya que los valores aumentaron al final del almacenamiento al obtener valores de hasta $29.00 \%$ (cuadro 3 ). 
Cuadro 3. Comparación de medias de Tukey ( $\mathrm{p}>0.05)$ para las variables evaluadas en semillas de cortadillo (Nolina cespitifera Trel.) en cinco muestreos bajo diferentes humedades relativas.

\begin{tabular}{|c|c|c|c|c|c|c|c|}
\hline Días & HR \% & HS & IVG & PN & PA & SM & SD \\
\hline \multirow{4}{*}{0} & 60 & $12.61 \mathrm{ef}$ & $2.66 \mathrm{a}$ & $80.00 \mathrm{a}$ & $5.00 \mathrm{ab}$ & $10.00 \mathrm{~d}$ & $5.00 \mathrm{e}$ \\
\hline & 75 & $12.61 \mathrm{ef}$ & $2.66 \mathrm{a}$ & $80.00 \mathrm{a}$ & $5.00 \mathrm{ab}$ & $10.00 \mathrm{~d}$ & $5.00 \mathrm{e}$ \\
\hline & 80 & 12.61 ef & $2.66 \mathrm{a}$ & $80.00 \mathrm{a}$ & $5.00 \mathrm{ab}$ & $10.00 \mathrm{~d}$ & $5.00 \mathrm{e}$ \\
\hline & 85 & $12.61 \mathrm{ef}$ & $2.66 \mathrm{a}$ & $80.00 \mathrm{a}$ & $5.00 \mathrm{ab}$ & $10.00 \mathrm{~d}$ & $5.00 \mathrm{e}$ \\
\hline \multirow{4}{*}{15} & 60 & $8.31 \mathrm{~g}$ & $2.51 \mathrm{ab}$ & $74.755 \mathrm{ab}$ & $7.18 \mathrm{ab}$ & $10.41 \mathrm{~d}$ & $7.64 \mathrm{de}$ \\
\hline & 75 & $12.40 \mathrm{ef}$ & $2.27 \mathrm{abc}$ & $67.16 \mathrm{abc}$ & $8.89 \mathrm{ab}$ & $12.30 \mathrm{~d}$ & $11.63 \mathrm{cde}$ \\
\hline & 80 & $13.90 \mathrm{cde}$ & $2.15 \mathrm{abcd}$ & $63.38 \mathrm{abcd}$ & $8.78 \mathrm{ab}$ & $12.54 \mathrm{~d}$ & $15.28 \mathrm{bcde}$ \\
\hline & 85 & $16.55 \mathrm{abc}$ & 2.01 bcde & 59.44 bcde & $7.22 \mathrm{ab}$ & $18.33 \mathrm{~cd}$ & $15.00 \mathrm{bcde}$ \\
\hline \multirow{4}{*}{30} & 60 & $9.16 \mathrm{~g}$ & $1.94 \mathrm{cde}$ & $57.61 \mathrm{cde}$ & $6.19 \mathrm{ab}$ & $17.26 \mathrm{~cd}$ & $18.92 \mathrm{abcd}$ \\
\hline & 75 & $14.09 \mathrm{cde}$ & 1.98 bcde & 60.00 bcde & $2.22 \mathrm{~b}$ & $18.88 \mathrm{~cd}$ & $18.88 \mathrm{abcd}$ \\
\hline & 80 & $13.81 \mathrm{de}$ & $1.81 \mathrm{cdef}$ & $54.23 \mathrm{cdef}$ & $4.83 \mathrm{ab}$ & $22.38 \mathrm{~cd}$ & $18.55 \mathrm{abcd}$ \\
\hline & 85 & $16.53 \mathrm{abc}$ & $1.69 \mathrm{defgh}$ & 50.27 defgh & $5.42 \mathrm{ab}$ & $17.92 \mathrm{~cd}$ & $26.38 \mathrm{ab}$ \\
\hline \multirow{4}{*}{60} & 60 & $10.16 \mathrm{fg}$ & 2.01 bcde & 59.68 bcde & $7.11 \mathrm{ab}$ & $14.93 \mathrm{~d}$ & $18.26 \mathrm{abcd}$ \\
\hline & 75 & $13.48 \mathrm{e}$ & 1.53 efghi & 44.44 efghi & $8.33 \mathrm{ab}$ & $30.55 \mathrm{bc}$ & $16.66 \mathrm{bcde}$ \\
\hline & 80 & 14.79 bcde & 1.34 fghij & 38.15 fghij & $11.56 \mathrm{a}$ & $29.27 \mathrm{bc}$ & $21.00 \mathrm{abc}$ \\
\hline & 85 & $16.39 \mathrm{abcd}$ & $1.22 \mathrm{hij}$ & $34.60 \mathrm{hij}$ & $10.12 \mathrm{ab}$ & $39.04 \mathrm{ab}$ & 16.23 bcde \\
\hline \multirow{4}{*}{90} & 60 & $10.18 \mathrm{fg}$ & $1.78 \mathrm{cdefg}$ & 51.55 cdefg & $11.08 \mathrm{a}$ & $20.71 \mathrm{~cd}$ & $16.64 \mathrm{bcde}$ \\
\hline & 75 & $13.37 \mathrm{e}$ & 1.27 ghij & 36.66 ghij & $7.78 \mathrm{ab}$ & $30.55 \mathrm{bc}$ & $25.00 \mathrm{ab}$ \\
\hline & 80 & $17.01 \mathrm{ab}$ & $1.06 \mathrm{ij}$ & $29.16 \mathrm{ij}$ & $11.23 \mathrm{a}$ & $30.59 \mathrm{bc}$ & $29.00 \mathrm{a}$ \\
\hline & 85 & $18.05^{\mathrm{a}}$ & $0.81 \mathrm{j}$ & $22.94 \mathrm{j}$ & $7.78 \mathrm{ab}$ & $49.37 \mathrm{a}$ & $19.90 \mathrm{abc}$ \\
\hline
\end{tabular}

Las medias con la misma letra indican que no son significativamente diferentes, HS=Humedad de la semilla, IVG $=$ Índice de velocidad de germinación, $\mathrm{PN}=$ plántulas normales, $\mathrm{PA}=$ plántulas anormales, $\mathrm{SM}=$ semillas muertas, $\mathrm{SD}$ $=$ semillas duras.

En la figura 4 se observa que IVG se mantuvo cuando las semillas fueron almacenadas a $60 \%$ HR a 5 y $15{ }^{\circ} \mathrm{C}$, y en condiciones de alta HR y temperatura alta mostraron los valores más bajos en las tres especies estudiadas. 

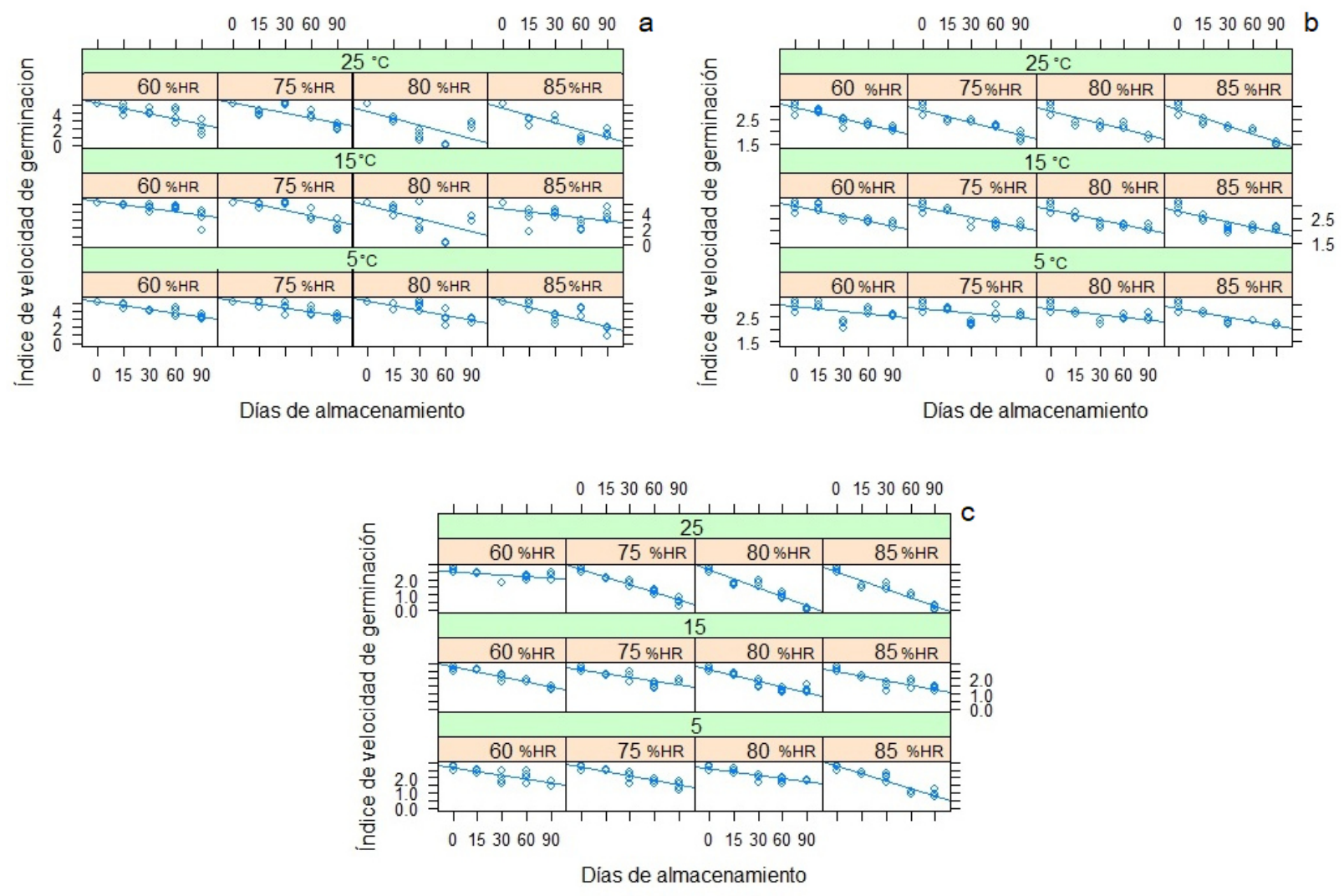

Fig. 4. Índice de velocidad de germinación (IVG) en semillas almacenadas a cuatro niveles de humedades relativa, a tres temperaturas, durante 90 días, a) Agave lecheguilla Torr, b) Lippia graveolens Kunt., c) Nolina cespitifera Trel.

El porcentaje de plántulas normales (fig. 5) disminuye a medida que la humedad relativa aumenta, cuando las semillas fueron almacenadas a 60 y $75 \%$ de HR en temperaturas de 5,15 y $25{ }^{\circ} \mathrm{C}$ durante los 90 días, los porcentajes PN se mantuvieron entre 81 y $95 \%$, y cuando se almacenaron a 80 y $85 \%$ de humedad relativa en temperatura de $25^{\circ} \mathrm{C}$, los porcentajes de PN oscilaron entre 5.0 y $18.5 \%$, las tres especies en estudio mostraron el mismo comportamiento.

\section{DISCUSIÓN}

Uno de los preceptos de almacenamiento establecidos por Harrington (1973), indica que la humedad relativa es más importante que la temperatura para la conservación de semillas (Magnitskiy y Guido, 2007). Los resultados obtenidos en esta investigación coinciden con lo establecido en éste precepto de almacenamiento, ya que a medida que la humedad relativa aumentó la germinación fue menor en las tres especies evaluadas.

En la presente investigación, el contenido de humedad de la semilla en Agave lecheguilla Torr almacenadas a HR $60 \%$ osciló entre 9.37 y $11.59 \%$, Lippia graveolens Kunt entre 4.5 y $7.55 \%$ y en Nolina cespitifera entre 7.0 y $12.3 \%$, los cuales se encuentran entre los parámetros establecidos por Harrington (1973) que para llevar a cabo un almacenamiento seguro el contenido de humedad de la semilla no debe ser mayor al 12\%. Lezcano et al. (2007), encontraron que para el almacenamiento seguro de Leucaena leucocephala el contenido de humedad de la semilla debe estar entre 10 y $12 \%$. 

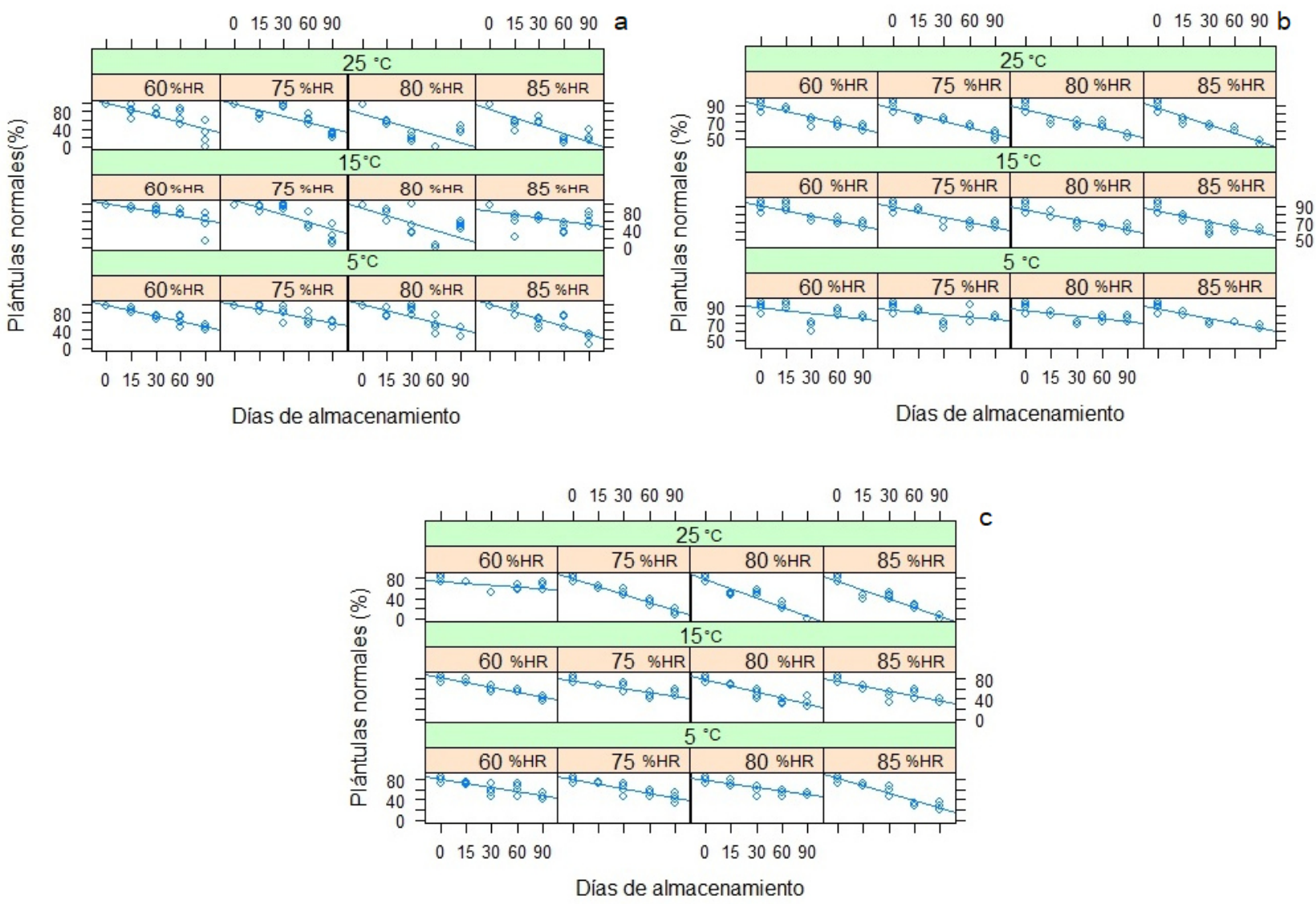

Fig. 5. Porcentaje de plántulas normales $(\mathrm{PN})$ en semillas almacenadas a cuatro niveles de humedades relativa, a tres temperaturas, durante 90 días, a) Agave lecheguilla Torr, b) Lippia graveolens Kunt., c) Nolina cespitifera Trel.

En un estudio realizado por Cardozo et al. (1999), encontraron que después de 24 meses de almacenamiento hubo un incremento en el contenido de humedad de la semilla y una reducción en la germinación de 6.9 a 7.4\% para Cucurbita moschata Duchesne ex Lam entre 5.9 y $6.41 \%$ para Arachis pintoi de 4.5 a 5.9\% para Phaseolus vulgaris var. Sangretoro y entre 6.6 a $6.9 \%$, resultados que son aproximados a los del presente estudio, después de 90 días de almacenamiento para Agave lecheguilla Torr, ya que cuando alcanzó 21.9\% de humedad en la semilla, PN disminuyó hasta 5.0\%; en semillas de Lippia graveolens Kunt cuando el contenido de humedad de la semilla fue $13.5 \%$ PN bajo a $47 \%$ y en semillas de Nolina cespitifera el valor máximo de contenido de humedad de la semilla fue de $21.8 \%$ con PN de $8.2 \%$.

González (2003), al realizar pruebas de germinación de semillas almacenadas de Bauhinia purpurea, observó que en ambientes con humedades relativas y temperaturas bajas, la germinación se mantuvo a niveles altos y cuando la humedad relativa aumentó a 20\% la germinación disminuyó de forma drástica a través del tiempo, resultados que coinciden con esta investigación, en las tres especies en estudio Agave lecheguilla Torr., Lippia graveolens Kunt., y Nolina cespitifera Trel., en humedades relativas de 80 y $85 \%$ se encontraron valores PN hasta un $5 \%$ a los 90 días de almacenamiento. 
Las semillas son higroscópicas por que absorben o liberan humedad, dependiendo del ambiente donde se encuentren y su contenido de humedad final y se estabiliza cuando estas se exponen a un ambiente específico, por un periodo de tiempo determinado, lo cual se conoce como humedad de equilibrio, depende además del tipo de semillas, la temperatura y humedad relativa del aire circundante (González et al., 2012).

El contenido de humedad de la semilla también se incrementa cuando aumenta la temperatura, siempre y cuando la humedad relativa permanezca estable, pero cuando la temperatura del aire se calienta, las semillas disminuyen su humedad de equilibrio (Montes et al., 2009).

Los resultados de humedad de equilibrio alcanzado por semillas de Agave lecheguilla Torr., Lippia graveolens Kunt., y Nolina cespitifera Trel., se vieron afectados por la temperatura aun cuando la humedad relativa se mantuvo constante. En estudio realizado por Alzugaray et al. (2007) en semillas de arroz en un ambiente con $70 \%$ de humedad relativa y temperatura de 15 ${ }^{\circ} \mathrm{C}$, la humedad en equilibrio fue de $13.8 \%$, pero al aumentar la temperatura a $25^{\circ} \mathrm{C}$ a la misma humedad relativa, la capacidad de retención de agua de ese ambiente aumentó y la humedad de equilibrio de la semilla en ese ambiente disminuyó a $13.3 \%$.

El contenido de humedad de la semilla, la temperatura y humedad relativa alta afectan especialmente la longevidad, aumentando la respiración y causando pudrición de las mismas (Aguirre, 2018).

Cuando la humedad relativa del aire supera el 75\%, el contenido de humedad de las semillas se incrementa rápidamente, en ambientes secos donde la humedad relativa no sobrepasa ese límite, sus cambios afectan poco el contenido de humedad de las semillas (Romero, 2018). En semillas de Agave lecheguilla Torr., Lippia graveolens Kunt. y Nolina cespitifera Trel., que fueron almacenadas a $15^{\circ} \mathrm{C}$ en condiciones de baja humedad relativa (60 y 75\%), PN no disminuyó de manera significativa, esto coincide con lo encontrado por Tang et al. (2019) quienes reportaron que hasta los seis meses de almacenamiento las semillas de aliso (Alnus acuminata), solera (Cordia gerascanthus) y cedrillo (Guarea guidonia) mantuvieron constante el porcentaje de germinación cuando se conservaron a $12^{\circ} \mathrm{C}$.

\section{CONCLUSIONES}

La humedad en equilibrio es fuertemente influida por las condiciones de almacenamiento para Agave lecheguilla Torr., Lippia graveolens Kunt. y Nolina cespitifera Trel.

Ambientes de alta humedad relativa y temperatura ocasionan una humedad de equilibrio alta, afectando de manera negativa la calidad fisiológica de las semillas de Agave lecheguilla Torr., Lippia graveolens Kunt., y Nolina cespitifera Trel.

En semillas forestales de zonas áridas pueden ser almacenadas en condiciones frescas y secas $60-75 \%$ de humedad relativa y $5-15^{\circ} \mathrm{C}$, sin que se vea afectada su capacidad germinativa.

Para las especies en estudio, almacenarlas bajo condiciones de alta humedad relativa $80-85 \%$, tiene como respuesta el aceleramiento del deterioro.

\section{LITERATURA CITADA}

Aguirre, R. 2018. Efecto de la humedad en el almacenamiento hermético a corto plazo de semillas de frijol (Phaseolus vulgaris). Agronomía Mesoamericana, I, 35. https://doi.org/10.15517/am.v1i0.25323

Alzugaray, C., Carnevale, N. J., Salinas, A. R., y Pioli, R. 2007. Factores bióticos y abióticos 
que afectan la calidad de las semillas de Schinopsis balansae Engl. y Aspidosperma quebracho-blanco Schltdl. Revista Iberoamericana de Micologia, 24(2), 142-147. https://doi.org/10.1016/S1130-1406(07)70030-X

Baskin C. y J. M. Baskin. 2004 Seeds: Ecology, Biogeography, and Evolution of Dormancy and Germination (A. Press, Ed.). San Diego and London.

Cardozo, C. I., López, Y., y Guevara, C. 1999. Estudio de deterioro de semilla en condiciones controladas de conservación. Acta Agronómica, 51(3-4), 89-101. Retrieved from http://www.revistas.unal.edu.co/index.php/acta_agronomica/article/view/47785

Doria, J. 2010. Generalidades sobre las semillas: su producción, conservación y almacenamiento. Cultivos Tropicales, 31(1), 74-85.

Gonzalez, F. 2012. Las zonas áridas y semiáridas de México y su vegetación (primera; S. de M. A. y R. N. (Semarnat), B. A. R. C. 4209. C. J. en la Montaña, D. F. C.P. 14210. Delegación Tlalpan, México, \& Www.semarnat.gob.mx, Eds.). Instituto Nacional de Ecología (INE-Semarnat) Periférico sur 5000, colonia Insurgentes Cuicuilco, C.P. 04530. México, D.F. www.ine.gob.mx.

González, J. D., Benítez, B., y Soto, F. 2012. Influencia De Diferentes Métodos De Conservación En La Germinación De Semillas De Palma Areca. 33(2), 56-60. Retrieved from http://scielo.sld.cu/scielo.php?script=sci_arttext\&pid=S0258$59362012000200008 \& \operatorname{lng}=$ en\&tlng=en

González, Y. 2003. Comportamiento germinativo y deterioro de las semillas de Bauhinia purpurea almacenadas al ambiente. pastos y forrajes, 26(2), 2000-2003.

Harrington, J. F. 1973. Problems of seed storage. W. Seed Ecology. Butterworth and Co., 251263.

Iriondo, J. M. 2001. Conservación de germoplasma de especies raras y amenazadas (Revisión). Invest. Agr.: Prod. Prot. Veg., 16(1), 10-11.

ISTA. 2014. "International rules for seed testing". International Seed Testing Association (ISTA).

Lezcano, J. C., Navarro, M., González, Y., y Alonso, O. 2007. Determinación de la calidad de las semillas de Leucaena leucocephala cv. Perú almacenadas al ambiente. Pastos y Forrajes. 30(4): 437-447

Magnitskiy, S., y Guido, A. 2007. Fisiología de semillas recalcitrantes de árboles. Agronomía Colombiana, 25(1): 96-103.

Mahecha Godoy, J. C. 2011. Determinación de los parámetros para la simulación matemática del proceso de deshidratación de la uchuva (Physalis Peruviana L.). 150. Retrieved from http://www.bdigital.unal.edu.co/4309/

Montes, E., Torres, R., Andrade, R., Pérez, O., Marimon, J., y Meza, I. 2009. Modelado de las isotermas de desorción de ñame (Dioscorea rotundata). Dyna 157, 157(2009): 145152.

RCT. 2017. R: A language and environment for statistical compiting. $R$ Foundation for Statistical Computing. Retrieved from http://www.r-project.org/

Romero-Saritama, J.M. 2018. Seed conservation: An alternative to store germplasm and recover threatened Ecuadorian forests. Neotropical Biology and Conservation, 13(1), 74-85. https://doi.org/10.4013/nbc.2018.131.09

Romero-Saritama, J.M., y Pérez-Ruíz, C. 2016. Rasgos morfológicos de semillas y su implicación en la conservación ex situ de especies leñosas en los bosques secos tumbesinos. Revista Ecosistemas, 25(2), 59-65. https://doi.org/10.7818/RE.2014.252.00

Sáenz R., J. T., Muñoz F., H. J., \& Rueda S. A. 2011. Especies Promisorias de Clima Templado Para Plantaciones Forestales Comerciales en Michoacán. Libro Técnico Núm. 10. Retrieved from http://biblioteca.inifap.gob.mx:8080/xmlui/bitstream/handle/123456789/3031/Especies promisorias de clima templado para plantaciones forestales comerciales en Michoacán_J. Trinidad Saenz Reyes.pdf?sequence $=1$

Salazar, J. A. C., González, J. M. P., Garay, Ó. J. A., González, V. A., y Peña, A. 2011. Adaptación de un modelo de deterioro a semillas de tomate de cáscara. Adaptation of a 


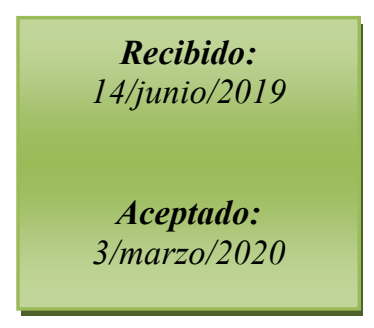

model to evaluate seed deterioration in husk tomato. Revista Fitotecnia Mexicana, 34(1), 53-61. Retrieved from http://www.scielo.org.mx/scielo.php?script=sci_arttext\&pid=S0187$73802011000100009 \& \operatorname{lng}=$ es\&nrm=iso

Tang, Y., Zhang, K., Zhang, Y., y Tao, J. 2019. Dormancy-breaking and germination requirements for seeds of Sorbus alnifolia (Siebold \& Zucc.) K.Koch (Rosaceae), a mesic forest tree with high ornamental potential. Forests, 10(4), 319. https://doi.org/10.3390/f10040319

Tropicos. 2019. "Missouri Botanical Garden." Retrieved from http://www.tropicos.org

Vega Gálvez, A., Lara Aravena, E., y Lemus Mondaca, R. 2006. Isotermas de adsorcion en harina de maiz. Ciencia Tecnología Alimentos, 26(4), 821-827. Retrieved from http://www.scielo.br/pdf/cta/v26n4/16.pdf

Winston, P. W. and Bates, D. H. 1960. Saturated solutions for the control of humidity in biological research. Ecology, 41(1), 232-237. 